\title{
VATE: VAlidation of high TEchnology based on large database analysis by learning machine
}

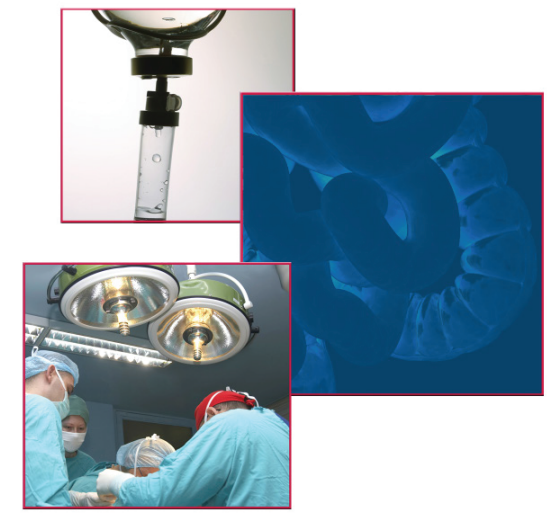

\author{
Elisa Meldolesi ${ }^{*, \ldots,}$, Johan van Soest ${ }^{2}$, Anna Rita Alitto', Rosa Autorino', \\ Nicola Dinapoli', Andre Dekker², Maria Antonietta Gambacorta', Roberto \\ Gatta', Luca Tagliaferri', Andrea Damiani' \& Vincenzo Valentini ${ }^{\ddagger}$,
}

\section{Practice points}

- Progress in individualized medicine has created new challenges.

- New technology implementation.

- Necessity to develop systems that allow shared decision making by the physicians and the patients and chose a tailored treatment.

- Standardization of the data collection - ontology.

- Sharing data: Semantic Web and Resource Description Framework.

- Statistical analysis.

- Privacy protection of individual patients.

- Development of predictive models based on individual patients features which complement existing consensus or guidelines.

SUMMARY The interaction between implementation of new technologies and different outcomes can allow a broad range of researches to be expanded. The purpose of this paper is to introduce the VAlidation of high TEchnology based on large database analysis by learning machine (VATE) project that aims to combine new technologies with outcomes related to rectal cancer in terms of tumor control and normal tissue sparing. Using automated computer bots and the knowledge for screening data it is possible to identify the factors that can mostly influence those outcomes. Population-based observational studies resulting from the linkage of different datasets will be conducted in order to develop predictive models that allow physicians to share decision with patients into a wider concept of tailored treatment.

Over the past decade, remarkable advances in cancer care with the adoption of newest diagnostic and treatment technologies has created new challenges [1].

The use and role of medical imaging technologies in clinical oncology has greatly expanded from a primarily diagnostic tool to award a central role in the context of individualized medicine. Multiple imaging features involving descriptors of intensity distribution, spatial relationships between the various intensity levels, texture heterogeneity patterns, descriptors of shape and the relations of the tumor with the surrounding tissues have been analyzed for their relationship with treatment outcomes or

\section{KEYWORDS}

- data mining • high technology $\bullet$ individualized medicine $\bullet$ large database - ontology $\bullet$ predictive model • Semantic Web 
gene expressions [2]. Beside these innovations, the concomitant research progress in pathology, biologic biomarkers (e.g., Kras, Braf, microsatellite instability, among others $[3,4])$, genomics and proteomics, justifying the growing trend toward 'individualized medicine' as key predictors for different treatment options (e.g., radiotherapy dose/fractionation and/or chemotherapy [CT]). Furthermore, tremendous advances in radiation therapy technology have allowed for remarkable precision in treatment delivery and for the realization of dose escalation with a concomitant decrease in treatment-related morbidity. Long considered to be a physical intervention, radiation therapy is now more accurately conceptualized as a biological intervention with effects at the cellular and molecular level, modulated through cellular signaling pathways and the immunological axis [5,6]. Accordingly, combinations of radiation therapy with targeted biological agents have proven growing efficacy and hold promise for future advances $[7,8]$. Therefore, several potential treatment options for each patient have to be considered instead of the inflexible 'one size fits all similar groups' approach. We have to move toward a 'shared decision making' process where an active interaction between doctors and patients results in the determination of the best therapeutic interventions.

In this context of progressive technologies and treatment innovation, a possible answer to the increasing necessity of individualized medicine is the development of predictive models allowing decision-making physicians to deliver tailored treatment; moving from an evidence-based treatment toward a personalized medicine concept (build on an evidence base) with an essential role for decision support systems (DSS). Predictive models based on individual patient features, complement existing consensus or guidelines and allow the transition from prescription by consensus to prescription by numbers.

The goal of this paper is to emphasize the influence of the interaction between the implementation of new technologies and different outcomes in radiation therapy, and to identify the factors that influence the outcomes (e.g., local recurrence, distant metastasis or overall survival). This identification will be performed using automated computer bots and the knowledge regarding screening and analysis of collected data. This approach can easily be reused and translated for a broad range of researches due to the usage of generalizable and flexible technologies in terms of data representation and semantics. The purpose of the VAlidation of high TEchnology based on large database analysis by learning machine (VATE) project is to address this analysis to the field of rectal cancer.

The advances in radiation oncology and the progress in individualized medicine have created, over the past decade, new challenges.

Personalized medicine is defined by the National Cancer Institute as "a form of medicine that uses information about a person's genes, proteins, and environment to prevent, diagnose, and treat disease. In cancer, personalized medicine uses specific information about a person's tumor to help diagnose, plan treatment, find out how well treatment is working, or make a prognosis" [9] .

To date, the standard efforts in the medical field and inherently also in oncology is to consider the outcomes of randomized clinical trials (RCTs) as having the key role in the definition of clinical guidelines, protocols and research. Besides RCTs, population-based observational studies are progressively emerging as a complementary form of research, especially to ensure that the result of clinical trials translate into tangible benefits in the general population [10]. In the past decades, the excellent internal validity of RCTs led to substantial improvements in treatment and outcome of patients with cancer. However, patients participating in RCTs are a selective subgroup of the general population, resulting in an inherent limiting factor when interpreting results, as the characteristics of a population seen in routine clinical practice is very different compared with a population included in RTCs [11]. Therefore, small benefits observed in highly selected trial patients are likely to disappear when the same treatments are applied in routine practice. Furthermore, some patient groups are underrepresented in RCTs, including the elderly, those with comorbidities $[12,13]$ and patients from under-represented ethnic and socioeconomic backgrounds [14-16]. Given the differences between patients recruited to trials and those seen in routine practice, increased toxicity might also be expected when the results of RCTs are applied to routine practice. Considering that, observational studies are essential to identify whether practice has changed appropriately, to document harms of therapy in a wider population, in patients of different age and with different comorbidities and to determine whether patients in routine practice are reaching the expected outcomes [17-19] with 
the expected toxicity. However, when multiples RCTs within one tumor type and comparing over decades and worldwide different treatment agents, classes and subpopulations, are observed in a pooled analysis [20-22], the best of both research regimens is reached: high amount of patient's variability with high data quality.

Although the findings of large randomized trials have addressed important questions, practical patient care issues remain that cannot be addressed by subgroup analyses of existing trials. Consensus guidelines reports try to help clinicians but still large areas of controversies exist [23-25].

Moving into the era of individualized medicine it is more and more important to develop systems that allow shared decision making by the physician and the patient and choose a tailored treatment. However, the development of DSS requires large heterogeneous datasets [1]. Features coming from many subdisciplines like diagnostic and clinical imaging, laboratory data, treatment outcome data, biologic environment, genomics and proteomics are routinely collected in the clinical practice and analyzed by innovative 'rapidlearning' research techniques. This interaction between clinical information and biomedical informatics driven research allows for extraction of knowledge of the masses for the benefit of the individual [26]. From the mathematical point of view, the development of predictive models requires a large amount of data to provide sufficient statistical power to act as an efficient and reliable predictive tool. Furthermore, a secondary dataset is needed for validation of the models, preferably by external (from a different institution) datasets [27]. Only after external validation a prediction model can be implemented as an acceptable decision support tool. Therefore, development of prediction models brings up some stringent and challenging demands on the quality as well as the quantity of the data. Hence, the necessity to create large databases realized by sharing and combining multiple datasets which are often horizontally (patients scattered across institutes) and vertically partitioned (features on one patient in separate data silos).

Considering that data could be shared among different departments of a single hospital or among institutes on a regional, national and international level, integration of information is a big challenge for data-sharing initiatives.

Furthermore, for some tumor types that most benefit from a radiation therapy treatment like rectal cancer, the integration of radiotherapy information and clinical data could also be highly significant for the development and validation of multifactorial prediction models. The process based on large databases approach, requires setting a flexible strategy for data collection, data mining and outcome reporting, which is quite different from the fixed design of a prospective randomized controlled trial. It is necessary to collect data without knowing beforehand what the relevant features will be and it implies to use tools to add new variables to the large databases in an ad-hoc manner. From a structural point of view, it is, first of all, necessary to proceed with a standardization of any considered variable in order to universally define data and procedures allowing automatic and consistent upload from any exploitable data source. This standardization process is obtained through the creation of an ontology, a terminological system where all the information, related in this case to rectal cancer, are specified and organized in a well-defined data collection model. At the same time, tailored data mining techniques should be selected to exploit the heterogeneity of the collected data, to get evidence from differences while accounting for confounding factors. Finally, an appropriate DSS should be finalized to provide practical support to clinical choices.

In this context the VATE project is growing. The term VATE derives from the Latin 'vates', in other words, prophet, fortune teller. The project, related to rectal cancer, was conceived from the idea that by combining the implementation of new technologies with clinical outcomes, it could be possible to give an answer to multiple controversial questions in radiotherapy. The development of predictive measures represents an important practical tool to support clinical choices besides the already existing consensus guidelines.

All the steps needed to define and organize relevant data, to link it between different Institutions (Figure 1) and to develop a predictive model with specific reference to the VATE project (Figure 2), are described in the following paragraphs.

\section{Standardized data collection \& ontology}

The process of standardization of the data collection will take advantage of an ontology. 'Ontology' is a compound word, composed of onto-, from the Greek ǒv $\tau$ ऽ (òntos) which is the present participle of the verb cijuí (eimi), in other 


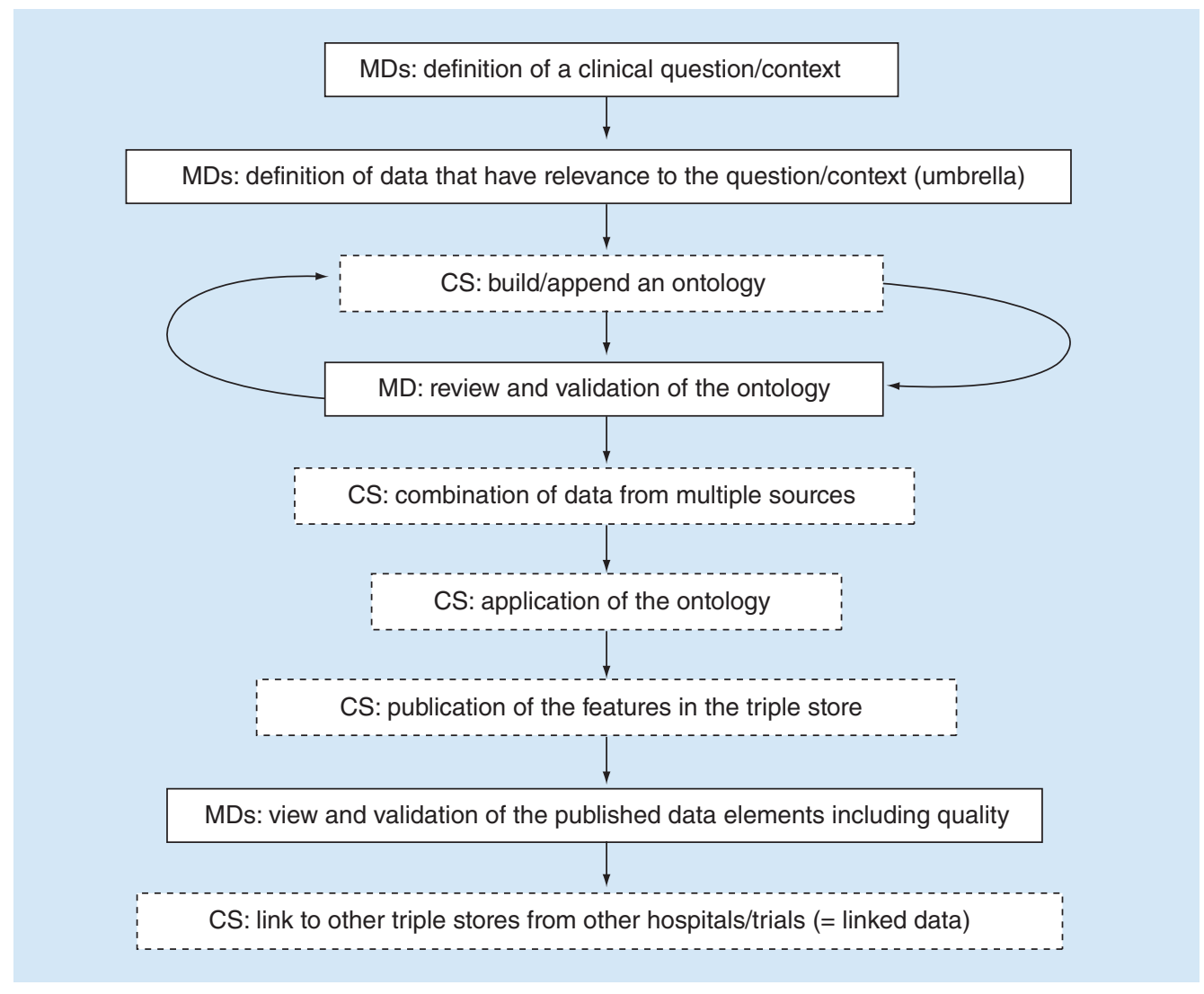

Figure 1. Steps needed to link data.

CS: Computer science; MD: Medical doctor.

words, 'to be, I am', and $\lambda$ ó $\gamma_{1}^{\prime} \alpha$ (lògia), in other words, 'science, study and theory'. Ontology formally represents knowledge as a set of concepts within a domain, and the relationships between those concepts. In practice, an ontology is (or can reuse) a classification system where each variable, in this case related to the domain of rectal cancer, can be represented using uniform and unambiguous definitions. Next to variable definitions, it can define relationships between variables. As these relationships can address variables defining space (e.g., relationships between institutional and standard terminologies) and time (e.g., versions of classifications), ontologies can enhance the understanding of datasets. Eventually, better and unambiguous understanding leads to an approach where rectal cancer research data could be made available without differences in interpretation; for now and the future. This kind of data collection model has to be open also to the necessity of extending the number of collectable variables over time to be able to comprehend all the clinical, treatment and technical advances.
A team of rectal cancer specialists with previous experience on handling prospective trials, data collection and contributing to the oncology registries, selected more than 200 features that they thought to have a relation to the outcome of rectal cancer patients. Features were organized according to three different tiers, with increasing granularity of data, to be able to answer different clinical questions. The first and most general level, the registry level, includes all the minimal information used for epidemiological analysis only. The second level, the Procedures level, includes treatment information, related toxicities and the evaluation of outcomes in term of disease-free survival and acute and late toxicities. The last tier, the research level, includes clinical and imaging information used for in-depth, advanced research projects only (Supplementary Material; see online at www.futuremedicine.com/ doi/full/10.2217/CRC.14.34). All the information needs to be converted to be semantically interoperable. Semantic interoperability (SIO) is important in the VATE project, as it is the 'glue' between different datasets. SIO means that 
data definitions and meaning of values are equal between different institutions and/or datasets. After making all datasets semantically interoperable, we are able to analyze and perform data mining on multiple datasets without compromising the outcome due to differences in definitions of variables between datasets. To achieve SIO, we used generally available and accepted coding systems (e.g., National Cancer Institute [NCI] Thesaurus, Common Terminology Criteria for Adverse Events [CTCAE], and Systematized Nomenclature of Medicine - Clinical Terms
[SNOMED]) to represent different datasets in a uniform manner. All needed concepts from the different coding systems are represented in our ontology. Relationships between concepts were determined after several architectural meetings, and are still under active development.

In detail, the registry level includes general patients' information starting from the location and/or type of the tumor according the ICD-10 classification; the treating hospital, age at the time of first rectal cancer diagnosis, gender and overall survival information of the patient.

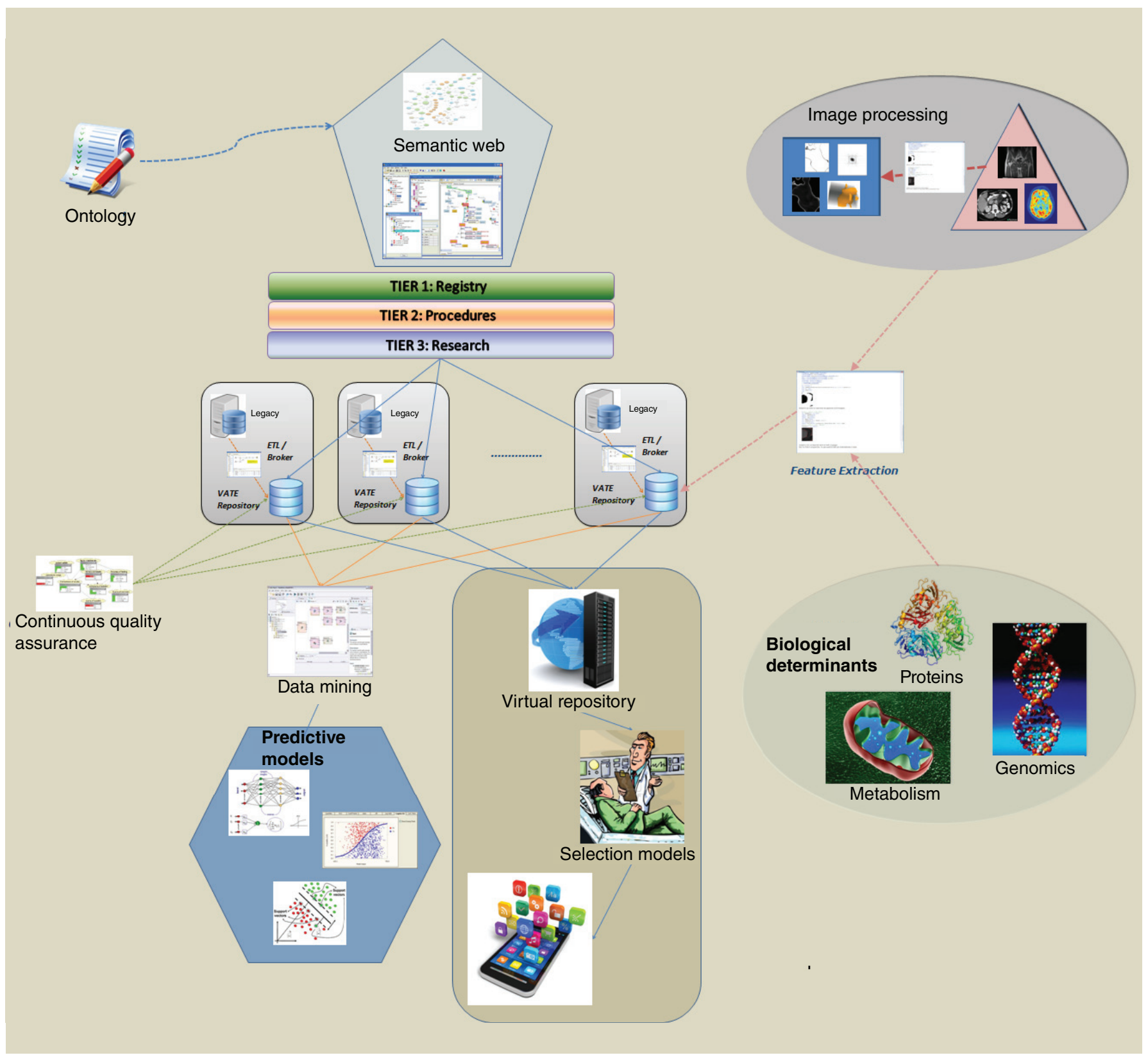

Figure 2. VAlidation of high TEchnology project infrastructure. 
The procedure level defines that all tumor and treatment information with related toxicities is recorded. The definition of the treatments' intent is stated. General patient characteristics are more detailed than the registry level and include height, weight, BMI, ethnicity, performance status, comorbidities and results of blood and serum test. Tumor characteristics are recorded at the diagnosis, after neoadjuvant treatment (if any) and after surgery. Tumor features include imaging characteristics used to stage the tumor such as its size, clinical TNM classification (cTNM; according to guidelines of American Joint Commission on Cancer) [28], Mesorectal Fascia status, presence/absence of extramesorectal lymph nodes, stage group [28], tumor location and methods of investigation, histological type (according to the International Classification of Disease for Oncology [ICD-O] classification [29]) and grade [28]. Intestinal margin, Tumor Regression Grading (TRG) score and residual tumor (R) are registered after surgery. In the procedure level all the rectal cancer treatment characteristics are recorded including (neo)adjuvant chemoradiotherapy information and surgery data. CT start and end date, CT agent, prescribed and delivered cycles and related toxicities are recorded. Radiotherapy (RT) on primary tumor $(\mathrm{T})$, lymph nodes $(\mathrm{N})$ and metastasis (M) information are described. Start RT date, total prescribed and delivered dose, fraction dose, fraction schedule, unexpected interruption days, RT technique, treatment position, immobilization devices, image-guided radiation therapy technique and frequency, intrafractional motion management devices, gross tumor volume (GTV) - Clinical Target Volume (CTV) and CTV-Pathological Target Volume (PTV) margins and related toxicities are recorded. Surgical intervention is described. Date of surgery, type of local and regional surgery, surgery technique and related toxicities are recorded. Type of stoma, stomas' placement and removal date, metastasis location - if any and metastasectomy are registered. Toxicity is recorded according to the CTCAE v3.0 or 4.0 classifications [30,31].

Finally, several outcome features are reported at this level. Imaging used for followup, date of followup, date of local recurrence - if any - date and location of metastasis $(\mathrm{M}+)$ - if any - and biopsy confirmation for local recurrence and/or $\mathrm{M}+$ are recorded.
The third and most detailed level, the research level, includes clinical and imaging information used for advanced research projects such as radiomics [32]. Concomitant medication, preexisting general and rectal quality of life $(\mathrm{QoL}$; EORTC QoL questionnaire [QLQ]-C30, EORTC QLQ-C29 and EQ-5D-5L) and tumor features including tumor markers are recorded. Diagnostic imaging (computed tomography, PET and/or magnetic resonance [MR]) for initial diagnosis and re-evaluation after neoadjuvant treatment - if any - are uploaded. Radiotherapy treatment planning information is stated. Planning computed tomography imaging, RTSTRUCT, RTPLAN, RTDOSE, RT algorithm, dose to OAR, QC in vivo dosimetry and QC patient specific pretreatment QA are recorded for radiotherapy treatment on T, N and $\mathrm{M}+$. Follow-up imaging information and FUP-general and rectal QoL evaluation are reported.

In the VATE project, a well-structured and continuously updated ontology available to all data providers is able to reduce data entropy. Using an ad-hoc manual data input interfaces and/or automated remapping applications (brokers) the ontology can be used to enforce SIO so that data can be shared between applications.

Data warehouses and connected medical software have to 'understand' what the exchanged data means in order to process it. Machines however are only able to deal with data and generate information by using terminologies, definitions and ontologies. Therefore, data has to be converted to information by putting it in context, generating knowledge by making sense of the information and comparing all the knowledge in the field to find the best path to take in the decision [33].

Finally, the usage of data warehouses containing data following terminological standards leads, in the VATE project, to the development of important tools (e.g., predictive models, treatment planning integrated software) able to provide practical support to clinical choices. By integrating radiotherapy treatment information (using a specific VATE application in the planning workstation) with clinical, biological and laboratory data, it will be possible to choose the best treatment option, the most convenient dose distribution or fractionation to be used for each individual patient treated for rectal cancer (Figure 3). 


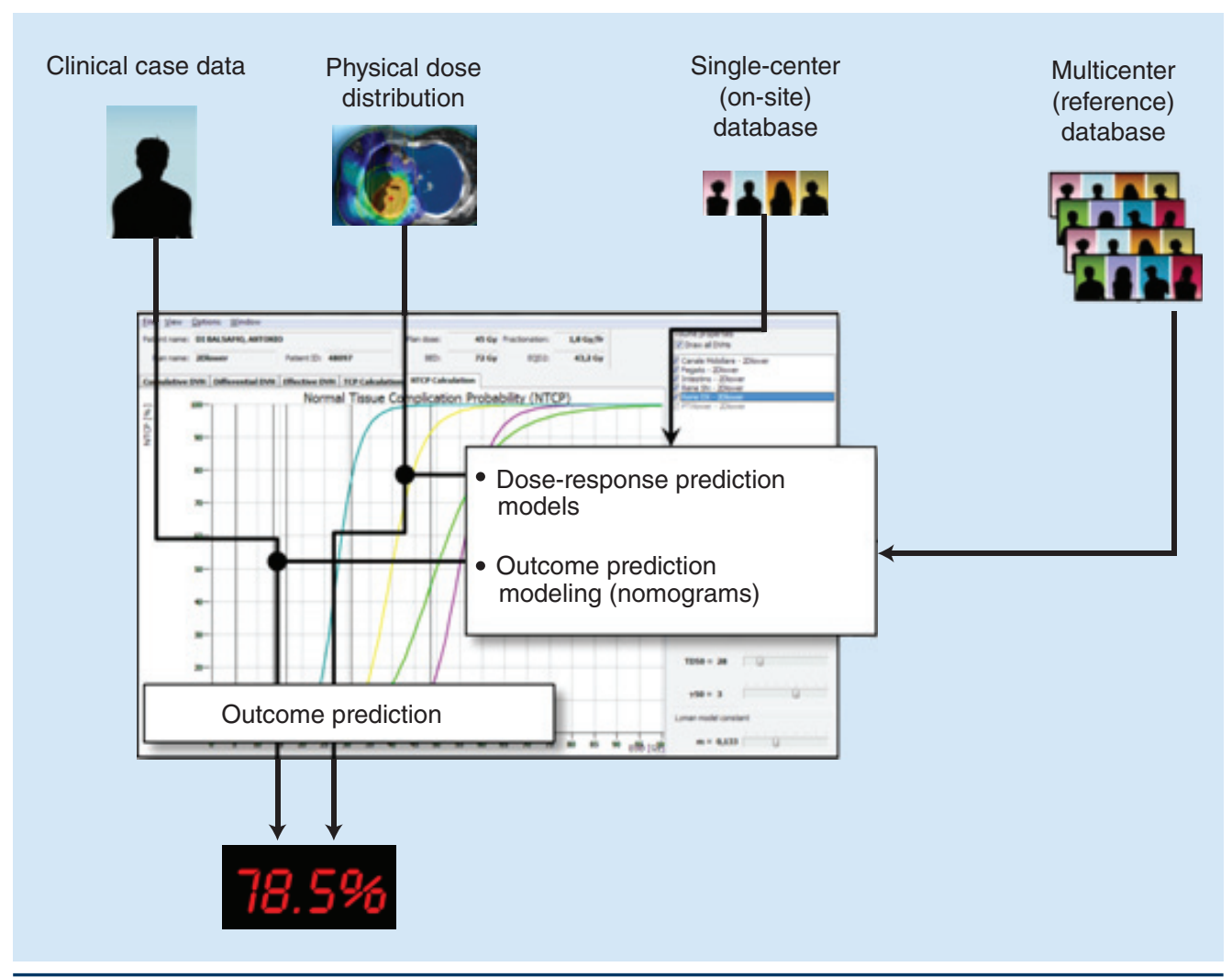

Figure 3. Example of VAlidation of high TEchnology database connection.

\section{Sharing data: data warehouse \& Semantic Web approaches}

The shift toward better electronic capture of patient records is a great opportunity to increase the portability and accessibility of patient information, with the promise to make data capture and processing easier and more reliable than in an ordinary handwritten paper medical record process. However, major challenges still exist when data needs to be shared among different departments of a single hospital or among institutes on a regional, national and international level. Besides overcoming legal and political barriers, SIO is a vital requirement [34]. In general, SIO is the ability of any communicating entity (not only computers) to share unambiguous meaning. For a computer network, this can be described as the ability of exchanging data reducing at a minimum (and eliminating in an ideal case) the entropy introduced in the information flow.

There are two basic approaches to use the ontology to share data. A proven but cumbersome method is the data warehouse, a novel and more flexible method is to use Semantic Web technology.
An example of data sharing infrastructure based on a data warehousing architecture was implemented between the Policlinico Universitario Agostino Gemelli in Rome, Italy (Gemelli) and the MAASTRO Clinic in Maastricht, The Netherlands in mid 2010 [35]. This infrastructure (Figure 4) has been used to share rectal cancer data by pooling two existing datasets from both institutes in order to facilitate research projects such as the Thunder clinical trial [36] and 'knowledge engineering' research $[37,38]$. Four categories of information were analyzed: clinical (e.g., demographics, TNM-stage, date of diagnosis and histopathology, among others); outcome (e.g., survival, local control and toxicity); imaging (e.g., diagnostic and follow-up PET, computed tomography and MR imaging) and treatment data from radiotherapy planning and delivery (e.g., delineation, planning-CT, dose matrix, beam setup, prescribed dose and fractions, cone beam CTs, Orthogonal EPID imaging and delivered fractions) and from nonradiotherapy treatments (e.g., surgery and CT). SNOMED Clinical Terms were used as the ontology base to convert Italian to English standard terms creating a new database (DB), the 


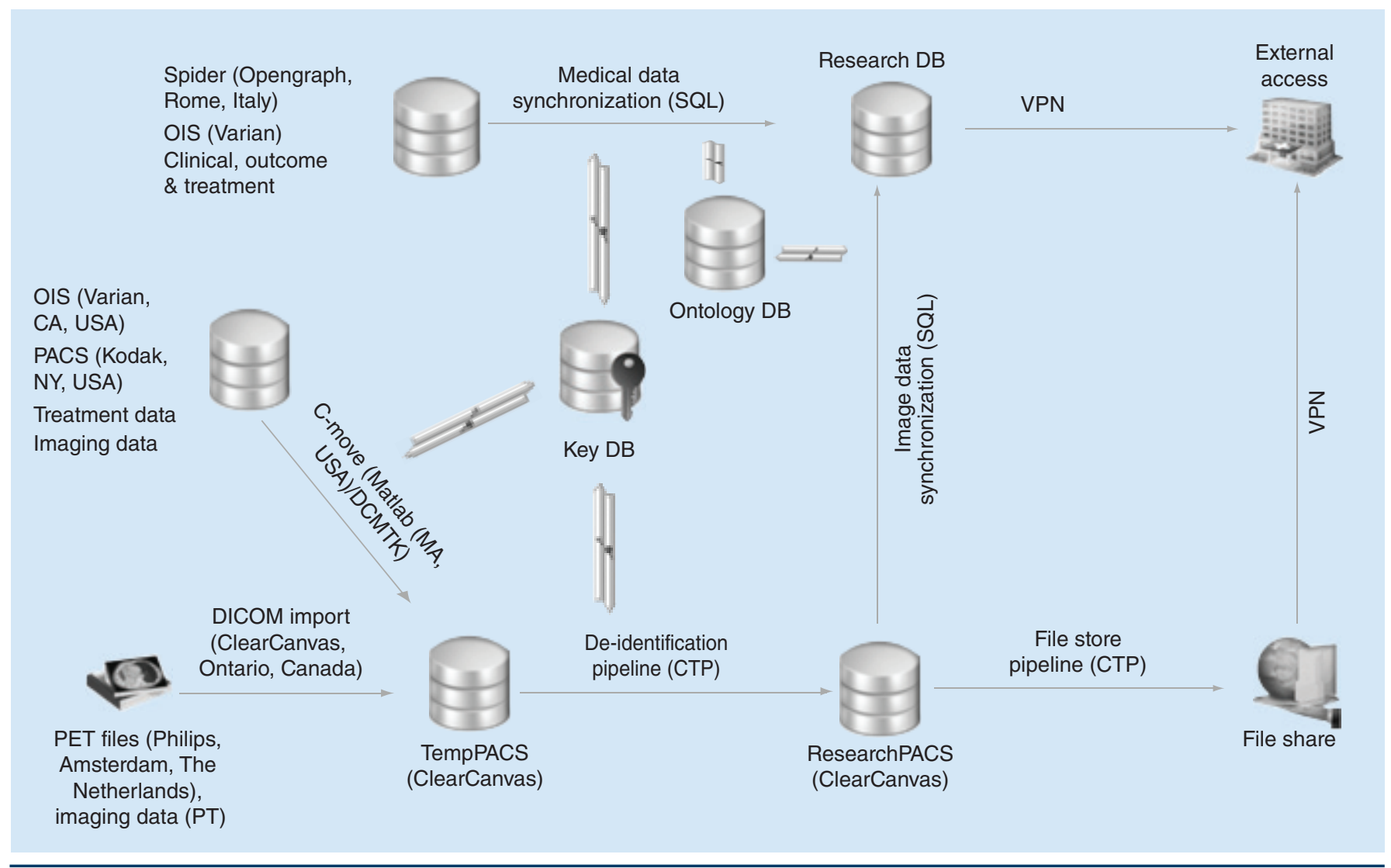

Figure 4. Overview of data sources, flow and external access.

CTP: Clinical Trial Processor; DB: Database; DCMTK: Digital Imaging and Communications in Medicine Toolkit; DICOM: Digital Imaging and Communications in Medicine; OIS: Oncology Information System; PACS: Picture archiving and communication system; SQL: Structured Query Language; VPN: Virtual private network.

Ontology DB, in which local terms were mapped to the SNOMED concept. Throughout a deidentification process (Key DB) and synchronization mechanism clinical and imaging data were linked together and a research database and a research picture archiving and communication system were created. Finally, a virtual private network connection was established to retrieve data from the research DB and research picture archiving and communication system. Using data mining and machine learning techniques, predictive models were developed [39].

The data warehouse approach results in a semantic interoperable dataset, which can be interpreted by applications. The main drawback of this solution is that a data warehouse is not very flexible and scalable for global implementation. If new data sources are added, specific data elements are added or if the data model is changed the interface to the data should be changed. Unfortunately this flexibility is essential for research, as research directions and thus the questions may change. If the data warehouse is changed, either the application to the data no longer works or everyone needs to continuously upgrade and update their local data warehouse to keep in line. This is undoable on a global scale. Fortunately, the current World Wide Web has proven to be scalable and very flexible; the Semantic Web is an extension of the World Wide Web ideas to expose semantic interoperable data.

For the Semantic Web technology, data is fundamentally represented using the Resource Description Framework (RDF) [40]. Data is represented in one table with three columns (subject, predicate and object). This RDF storage can be queried and modified using the SPARQL Protocol and RDF Query Language (SPARQL) [41]. In a typical setting, a SPARQL end point is placed on top of the RDF storage, which enables querying the RDF storage. SPARQL queries can query one or multiple (linked) triplets (one row of subject, predicate, object), based on the search criteria, and present the query results in an 
$\mathrm{n}$-by- $\mathrm{m}$ table (where $\mathrm{n}$ is the number of variables queried, and $\mathrm{m}$ is the number of rows returned). By representing the ontology using RDF (and subsequently Resource Description Framework Schema and Web Ontology Language [OWL] implementations of RDF), the Semantic Web technology enables a semantically interoperable representation of data elements defined in the umbrella protocol (Table 1).

Flexibility is an unavoidable requirement of the VATE project and finds in the Semantic Web technology the most efficient data sharing infrastructure. The power of the Semantic Web technology is the extremely flexible RDF representation but also the federated nature of the web where data and knowledge can reside anywhere and can be queried SPARQL [41]. As RDF contains a metastructure (one table with three columns), it is independent of domainspecific structures, which enables flexibility and federation. As an example the CTCAE system (grades and definition) is available in a linked data representation, meaning that this knowledge can be reused without the need to rerepresent this knowledge. The strategy taken in our protocol is to add SPARQL end points to local data sources (ranging from excel sheets or XML files to relational databases) as early as possible. This allows for internal (institutional) federated SPARQL queries to gather data for analysis. If this is not possible, an alternative strategy is to extract the data from the source (using 'classical' Extraction, Transformation, Loading [ETL] tools) and store the data in an RDF store. A further strategy is that all knowledge and data that can be made public (e.g., clinical trial data) or is already public can be accessed through a public SPARQL end point. Data that cannot be made public (e.g., routine clinical care data) will be available through private SPARQL end points.
Depending on the local regulations these private end points will be only available from inside the hospitals, or to authenticated and authorized users outside the hospital.

An example of a query using the Semantic Web platform [43], where values stored in the 'sexCode' and 'stageCode' fields follow a publicly encoded ontology (containing a thesaurus) established by the National Cancer Institute [44] has been created.

Finally, the possibility to share information between different institutions, as in the VATE project, allows getting data from the multicenter (reference) database. Therefore, the choice of the best treatment protocol for each single rectal cancer patient in terms of tumor control and normal tissue sparing could be simplified by the possibility to also use data stored on other radiotherapy treatment planning workstations (Figure 2).

\section{- Data quality issues \& proposed mitigation}

The infrastructure described above ensures that an application can get access to data at various institutes without the need for query rewriting or knowing the specifics of the underlying source systems. But while this infrastructure generalizes data extraction it does not guarantee the quality of the data from which application have to learn. To prevent a 'garbage in, garbage out' classification of the produced knowledge, the data quality must be known and if possible improved. The best way to improve the data is using the umbrella protocol to ensure that data is prospectively collected in the most consistent and best possible way. However, because resources are limited to be complete and correct in data collection and/or to reevaluate historical data, data quality issues will always be present. The expected data quality issues and the measures to

\section{Table 1. Examples of 'semantic' triple representation.}

\begin{tabular}{|llll}
$\begin{array}{l}\text { Subject } \\
\text { Patient }\end{array}$ & $\begin{array}{l}\text { Predicate } \\
\text { Has been diagnosed with }\end{array}$ & $\begin{array}{l}\text { Object example (URI) } \\
\text { Malignant neoplasm of rectum (http:// } \\
\text { purl.bioontology.org/ontology//CD10/C19) }\end{array}$ & ICD-10 \\
Patient & Has biological sex & $\begin{array}{l}\text { Male (http://ncicb.nci.nih.gov/xml/owl/ } \\
\text { EVS/Thesaurus.owl\#C20197) }\end{array}$ & NCI Thesaurus \\
& & $\begin{array}{l}\text { Female (http://ncicb.nci.nih.gov/xml/owl/ } \\
\text { EVS/Thesaurus.owl\#C16576) }\end{array}$ & \\
& & $\begin{array}{l}\text { T1 stage finding (http://ncicb.nci.nih.gov// } \\
\text { xml/owl/EVS/Thesaurus.owl\#C48720) }\end{array}$ & NCI Thesaurus \\
Disease & Has stage finding & & \\
& & & \\
\hline $\begin{array}{l}\text { ICD-10: 10th revision of the International Statistical Classification of Diseases and Related Health Problems; NCl: National Cancer } \\
\text { Institute; URI: Uniform resource identifier. } \\
\text { Reproduced with permission from [42]. }\end{array}$ & & \\
\hline
\end{tabular}


Table 2. VATE project: expected data quality issues and measures of mitigation.

\begin{tabular}{|c|c|c|c|}
\hline Problem & Example problem & Mitigation & Example mitigation \\
\hline $\begin{array}{l}\text { Completely } \\
\text { missing data }\end{array}$ & $\begin{array}{l}\text { Hospital A does not have a } \\
\text { PET scanner, so all PET derived } \\
\text { features are missing. Hospital B } \\
\text { does have and use a PET scanner } \\
\text { in all rectal cancer patients }\end{array}$ & $\begin{array}{l}\text { Impute based on populations from } \\
\text { other centers and what is known for } \\
\text { the patient }\end{array}$ & $\begin{array}{l}\text { Suppose a (probabilistic) relation between tumor } \\
\text { size and SUV is learned from Hospital B, then the } \\
\text { tumor size of Hospital A can be used to infer SUV } \\
\text { max in Hospital A even if they don't have a PET } \\
\text { scanner and are using the same scan protocols }\end{array}$ \\
\hline $\begin{array}{l}\text { Randomly } \\
\text { missing data }\end{array}$ & $\begin{array}{l}\text { Physician in Hospital A forgets } \\
\text { to note the TNM stage of the } \\
\text { patient }\end{array}$ & $\begin{array}{l}\text { Because data are missing randomly, } \\
\text { the percentage of missing data is } \\
\text { generally low and samples are large, } \\
\text { machine learning techniques will be } \\
\text { unaffected by these errors }\end{array}$ & Do nothing \\
\hline $\begin{array}{l}\text { Biased data: } \\
\text { continuous }\end{array}$ & $\begin{array}{l}\text { A PET scanner is calibrated } \\
\text { differently in hospital A than in } \\
\text { hospital B, so the SUV values are } \\
\text { different. Hospital B uses the SOP } \\
\text { of the umbrella protocol }\end{array}$ & $\begin{array}{l}\text { Assuming patients are similar a } \\
\text { conversion is possibly between two } \\
\text { distributions }\end{array}$ & $\begin{array}{l}\text { Determine the distribution of SUV values in hospital } \\
A \text { and B and derive a conversion function from SUVS } \\
\text { in hospital A to hospital B }\end{array}$ \\
\hline $\begin{array}{l}\text { Biased data: } \\
\text { scoring } \\
\text { system }\end{array}$ & $\begin{array}{l}\text { CTCAE v3 was used, but after } \\
\text { data X CTCAE v4 was used to } \\
\text { score toxicities }\end{array}$ & $\begin{array}{l}\text { Impute the new score from the old } \\
\text { score, if possible }\end{array}$ & $\begin{array}{l}\text { A (probabilistic/deterministic) conversion between } \\
\text { the two CTC systems is possible }\end{array}$ \\
\hline $\begin{array}{l}\text { Randomly } \\
\text { errors }\end{array}$ & $\begin{array}{l}\text { In hospital A, a physician has } \\
\text { noted an incorrect stage on an } \\
\text { individual patient }\end{array}$ & $\begin{array}{l}\text { Because errors are random, the } \\
\text { percentage errors will be low and } \\
\text { samples are large, the effect when } \\
\text { using machine learning will be low }\end{array}$ & Do nothing \\
\hline $\begin{array}{l}\text { Biased } \\
\text { missing data }\end{array}$ & $\begin{array}{l}\text { In hospital A, severe toxicities are } \\
\text { noted but mild toxicities are not. } \\
\text { In hospital B toxicities are always } \\
\text { noted }\end{array}$ & & $\begin{array}{l}\text { Compare occurrence of toxicities in hospital A } \\
\text { with hospital B. Detect too low, unexplained mild } \\
\text { toxicities in hospital A. Infer a probability of mild } \\
\text { toxicity for patients of hospital A based on the } \\
\text { distribution of hospital B }\end{array}$ \\
\hline
\end{tabular}

mitigate them in the VATE project are described in Table 2.

As can be seen from Table 1, many of the mitigation strategies assume that there is a hospital (or maybe a clinical trial) that does have high quality data which can be used to detect and correct any quality issues in the low quality hospital. As the volume of data increases, these mitigations are expected to become easier and better.

A number of strategies are employed to detect data quality problems. First and foremost is the domain knowledge from the physicians, physicists etc. that are involved in the project, they can often name many of the data quality issues that can be expected before they are observed. Another strategy is to include the treating hospital as a feature during learning. If the machine learning algorithm selects the treating hospital as predictive for outcome then data quality is suspected.

In the VATE project the imputation method used to leverage the data from the high quality source will be based on Bayesian statistics (e.g., Bayesian networks) as these have been shown to be the best for imputation $[37,45]$.

\section{Statistical analysis}

The strategy to collect data in a standard and consistent manner and to analyze them properly for decision support is named 'Umbrella protocol' [46]. The main difference between the development of a regular protocol and an umbrella protocol is the necessity, in the former to predefine the hypotheses investigated. In an Umbrella protocol multiple hypotheses are considered but none are predefined.

The aim of the VATE project is not only to develop, validate and improve prediction models for overall survival or for acute and late radiation-induced side effects for rectal cancer patients, but also to use the prediction models to better inform patients on the risks (acute and late toxicity) and benefits (overall survival) of the treatment; going toward an individualized treatment. Furthermore, the realization of software (VATE software) usable during planning procedures, able to provide to clinicians reliable 
models to predict the outcome both for tumor control and the probability to develop complications, will provide the possibility to optimize internal guidelines for choosing treatment protocols and evaluate case by case the best technology, dose distribution or fractionation to be used for an individual patient's treatment plan. Finally, emerging radiation delivery techniques or other new diagnostic or treatment options can be investigated to assess their added value using the distributed machine learning (and data mining) strategy. Eventually, this strategy would allow us to compare the outcome of new treatment options clinically introduced, with the current standard (and accompanying retrospective data) in terms of radiation-induced toxicity, patient-rated symptoms and $\mathrm{QoL}$ and overall survival.

To learn from the collected data, a distributed learning approach will be taken in the VATE project. The aim of distributed learning is to learn a model from the data without the need for data to leave the individual hospital. A distributed machine learning algorithm is split up into two parts: One master application which is installed on a central server (called the gateway) and coordinates the learning between the hospitals. The second part is a local learning application which is installed at each hospital. It has access to the local data and performs learning tasks but does not share patient data with the outside world. The local application learns a model from local data. This local model is sent to the gateway where it is compared with the models from the other hospitals. A consensus model is generated and sent back to the hospitals for refinement. After preset convergence criteria are met, a final consensus model is created. This method works for a variety of models as described in literature [47].

The information exchanged between gateway and local nodes is limited to aggregated values (e.g., parameter weights, general statistics and coefficients) and contains no patient data. All traffic between gateway and local nodes is managed, monitored and audited by the infrastructure. An entire learning run is an iterative process that usually requires many cycles $(\sim 500)$ until the master determines that the learning process has been completed (see Figure 5 ).

The width of dataset used in VATE project has to be considered as the first pitfall to address correct statistical and data analysis tools in order to get reliable results and models. Indeed the generic definition of 'large database' links itself with many technical issues that have to be correctly implemented in order to get an effective analysis of the data. We can consider two large families of data analysis tools: inferential regression analysis tools, mainly based on the relationship between outcomes (binary, continuous or multinomial) and covariates, or elements in the dataset, that establish a data-to-outcome oneway link, investigated using traditional statistical tools as linear models, generalized linear models, survival models, among others; machine learning analysis tools, used creating a recursive relationship between outcomes and generating data, with a complex automation background, that can resolve complex relationships between elements in the dataset and final results, too complex, in some situations, to be investigated by using the tools of the family 1 . Each model, anyhow defined, must undergo to a strict evaluation process mainly based on internal and external validation [48] in order to become a reliable tool to be used in clinical contexts.

The methodological process to learn, in other words, to go from data to useful decision support is depicted in Figure 6 [1]. From the hypothesis, experts determine which features should be included in the learning effort. In the preprocessing step data quality is improved by imputation for missing data and outlier and bias detection and correction. Especially Bayesian approaches will be used as these are considered the best method [45]. Then the data is split into a training and a validation cohort. The training cohort is used in a feature selection and classification algorithm to train a model. The machine learning approaches can vary but are typically Bayesian networks [37,49], Support Vector Machines [50] or Cox regression [22]. The final model can be presented to the end-user in a variety of ways such as nomograms [22] or via interactive websites such as PredictCancer [39].

The performance of the models will be assessed in terms of discrimination as well as calibration. External validation cohorts will be used for this purpose. Discrimination will be assessed using the c-statistic or area under the curve of the receiver operating characteristic. The c-statistic is comparable to the area under the curve for dichotomous outcomes, but can also be used for Cox regression analysis. A graphical assessment of calibration will be done by plotting the expected versus the observed outcome. In addition, the Hosmer-Lemeshow test will be used. 


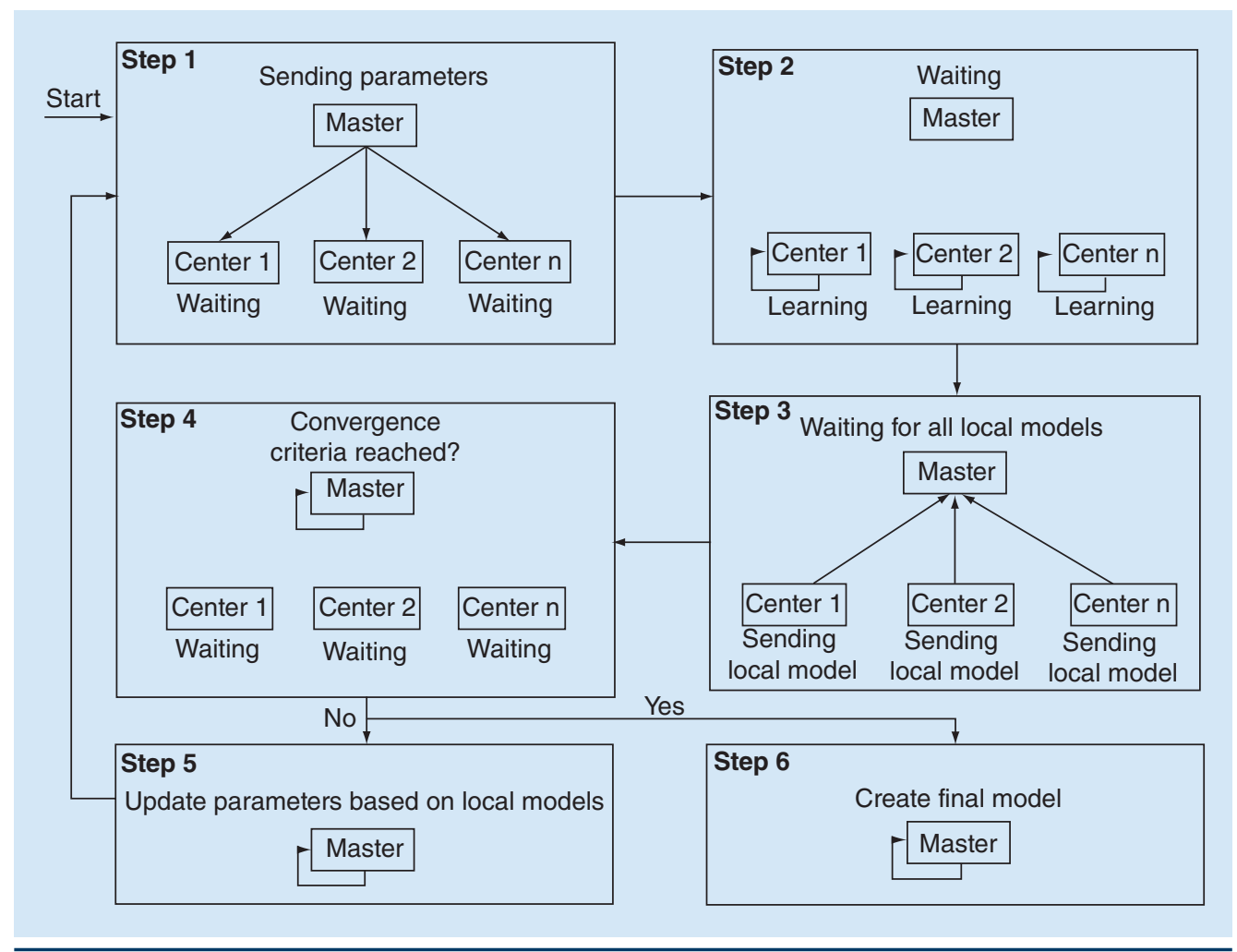

Figure 5. Distributed machine learning flow.

The clinical value of the models will be assessed using decision curve analysis [51,52]. This will make it possible to compare the clinical value of different models over a number of decision thresholds (or cut-off points for probability of outcome). Using this method, there is no necessity to choose an a priori cutoff point (for a clinical decision).

\section{Privacy protection of patients}

To ensure the privacy of individual patients, all data will be pseudonomized or anonymized. Within each institute participating to the VATE project consortium, a local data manager will be responsible for pseudo/anonymizing the patient data. All data, originating from local repositories at the institute's site, is routed to a local repository. During transfer, all data will pass software to anonymize/pseudonomize all identifiable and traceable information. When anonymizing data, information (not needed for research) is removed and will not enter the local repository; while pseudonymization replaces data with other values, based on a list of original and replaced values. As a consequence, from the local SPARQL end point, which is queried by other research group members from inside or outside the institute during normal activity, it is impossible to reconcile clinical information to the relevant patient.

A second layer of privacy protection is that data does not need to leave the institute in the process of distributed machine learning. This is possible as the central 'master' sends possible prediction models rather than fetching the data from remote nodes. Only statistical indexes totally unrelated to specific patients are exchanged between nodes and their master [47,53-54]. Although this protocol does not require an intervention and the data is fully deidentified, internal review board ethics approval is recommended before implementing a local node.

\section{Conclusion \& future perspective}

The interaction between the implementation of new technologies and different selected outcomes through the usage of automated computer bots and the knowledge for screening the collected data can allow a broad range of researches to be expanded, due to the very generalizable and flexible technology utilized.

In the field of oncology, the possibility to predict the outcome for a certain patient in combination with a specific treatment with more accuracy will lead to more specific risk groups and thus 


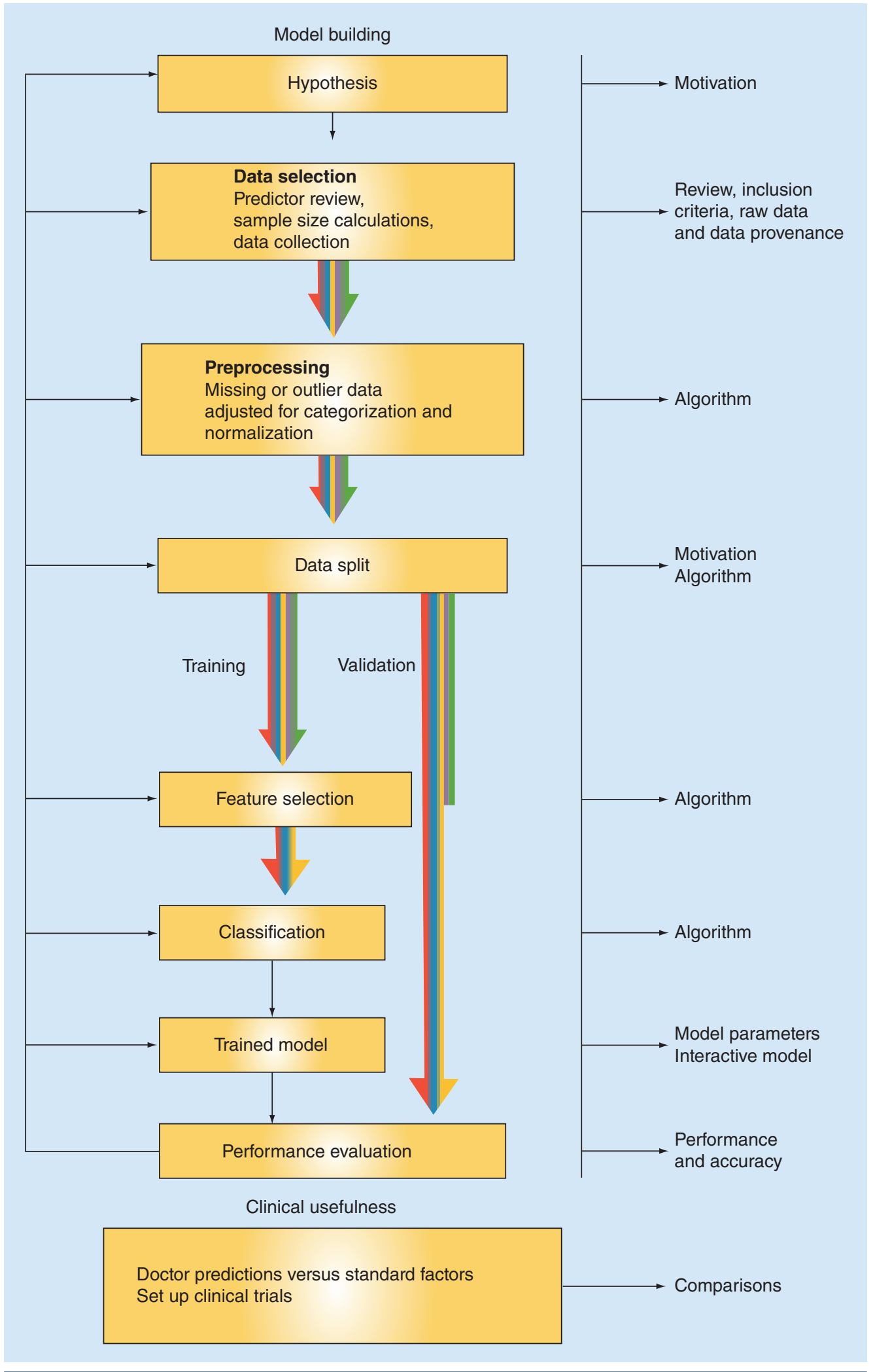

Figure 6. Schematic overview of methodological processes in clinical decision support system development.

Reproduced with permission from [1]. 
better treatment decisions for individual patients. It will also stimulate research focused on specific risk groups, trying to find new treatment options or other combinations of treatment options for these subgroups. Furthermore, it can be expected that treatment will be more personalized, which will not only save patients from unnecessary toxicity and inconvenience, but will also facilitate the choice of the most appropriate treatment. Currently, this choice is based on general guidelines resulted from large randomized trials that commonly include patients specifically selected to the research point. Complementary information coming from the combination of RCT and population-based observational studies will be able to find out new drugs and treatment strategies, and to review the efficiency of different approaches in large patients' populations.

The development of measures that allow the decision-making physician to deliver tailored treatment is particularly important as the oncology profession moves into the era of individualized medicine.

Clinicians are now facing two new challenges: one is the trend toward 'individualized medicine' trying to consider several potential options for each patient in place of inflexible 'one size fits all similar groups' approach. Second there is a move toward 'shared decision making', where doctors and patients actively discuss and decide on therapeutic interventions.

Therefore, predictive models, based on individual patient features, which complement existing consensus or guidelines allow us to move from prescription by consensus to prescription by numbers. The end results is a more patient specific selection from the treatment options menu and a possibility to share decisions with patients based on an objective evaluation of risks and benefits [55]. All of this comes with the 'overload' of information for physicians and patients. Therefore, it is important that a physician properly informs the patient on his or her options; patients might take the risk of specific inconveniences over a less aggressive treatment, or vice versa.

The development and validation of advanced predictive models based on large data collections is a necessary step toward the construction of a generation of software applications aimed to add a new dimension of knowledge during the planning phase. These tools will give clinicians a better view of the probability associated with the specific patient, both in terms of tumor control and of the development of complications. This will eventually lead to an optimization of the internal guidelines for choosing treatment protocols and evaluate case by case the best technology, dose distribution or fractionation to be used for the single, individualized treatment.

Finally, considering the important role that predictive models could play in the clinical practice, clinicians must be aware of the limits and confidence intervals of these prediction models. They need to be internally validated taking into account the quality of the collected data. An external validation of models is also essential to support general applicability of the prediction model. Therefore structural collaboration between different groups is crucial to generate enough anonimized large databases from patients included or not in clinical trials.

Prediction of outcome in order to choose the optimal treatment is complicated because of the very complex, dynamic nature of cancer and organs at risk. Therefore, treatment can only become more personalized if accurate, sciencebased decision aids are developed, that can offer assistance in clinical decision making in daily practice.

VATE project is trying to expand on global scale the outcomes that have been reached with previous studies $[22,50]$. This preliminary work is daily being used via predictcancer.org by physicians and patients so they find it useful. The clinical impact of the development and validation of advanced predictive models will evolve, over the next few years, to the possibility of having decision supporting tools that can help doctors in the daily practice.

The possibility to have a better view of the probability associated with the specific patient, will eventually lead to an optimization of the internal guidelines for choosing treatment protocols and evaluate case by case the best treatment strategy. In case of radiotherapy it could also be helpful in choosing the best technology, dose distribution or fractionation to be used for the single, individualized treatment.

\section{Financial \& competing interests disclosure}

The authors have no relevant affliations or financial involvement with any organization or entity with a financial interest in or financial conflict with the subject matter or materials discussed in the manuscript. This includes employment, consultancies, honoraria, stock ownership or options, expert testimony, grants or patents received or pending or royalties.

No writing assistance was utilized in the production of this manuscript. 


\section{References}

Papers of special note have been highlighted as: - of interest; $\bullet$ of considerable interest

1 Lambin P, van Stiphout RGPM, Starmans MHW et al. Predicting outcomes in radiation oncology - multifactorial decision support systems. Nat. Rev. Clin. Oncol. 10(1), 27-40 (2013).

- Explanation of the methodological process of the development of a decision support system.

2 Lambin P, Rios-Velazquez E, Leijenaar R et al. Radiomics: extracting more information from medical images using advanced feature analysis. Eur. J. Cancer 48(4), 441-446 (2012).

3 Yamane LS, Scapulatempo-Neto C, Alvarenga $\mathrm{L}$ et al. KRAS and BRAF mutations and MSI status in precursor lesions of colorectal cancer detected by colonoscopy. Oncol. Rep. doi:10.3892/or.2014.3338 (2014) (Epub ahead of print).

4 Lin C-C, Lin J-K, Lin T-C et al. The prognostic role of microsatellite instability, codon-specific KRAS, and BRAF mutations in colon cancer. J. Surg. Oncol. doi:10.1002/ jso.23675 (2014) (Epub ahead of print).

5 Bentzen SM. Preventing or reducing late side effects of radiation therapy: radiobiology meets molecular pathology. Nat. Rev. Cancer 6(9), 702-713 (2006).

6 Fowler JF. 21 years of biologically effective dose. Br. J. Radiol. 83(991), 554-568 (2010).

7 Glynne-Jones R, Hadaki M, Harrison M. The status of targeted agents in the setting of neoadjuvant radiation therapy in locally advanced rectal cancers. J. Gastrointest. Oncol. 4(3), 264-284 (2013).

8 Bonner JA, Harari PM, Giralt J et al. Radiotherapy plus cetuximab for locoregionally advanced head and neck cancer: 5-year survival data from a phase 3 randomised trial, and relation between cetuximab-induced rash and survival. Lancet Oncol. 11(1), 21-2 (2010).

9 NCI. NCI Dictionary of terms: personalized medicine.

www.cancer.gov/dictionary? cdrid $=561717$

10 Booth CM, Tannock IF. Randomised controlled trials and population-based observational research: partners in the evolution of medical evidence. Br. J. Cancer 110(3), 551-555 (2014).

- Good analysis of strengths and limitations of randomized clinical trials and populationbased observational researches.

11 Zietman AL. Falsification, fabrication, and plagiarism: the unholy trinity of scientific writing. Int. J. Radiat. Oncol. Biol. Phys. 87(2), 225-227 (2013).

12 Tyldesley S, Zhang-Salomons J, Groome PA et al. Association between age and the utilization of radiotherapy in Ontario. Int. J. Radiat. Oncol. Biol. Phys. 47(2), 469-480 (2000).

13 Faivre J, Lemmens VE, Quipourt V, Bouvier AM. Management and survival of colorectal cancer in the elderly in population-based studies. Eur. J. Cancer 43(15), 2279-2284 (2007).

14 Bach PB, Cramer LD, Warren JL, Begg CB. Racial differences in the treatment of early-stage lung cancer. $N$. Engl. J. Med. 341(16), 1198-1205 (1999).

15 Boyd C, Zhang-Salomons JY, Groome PA, Mackillop WJ. Associations between community income and cancer survival in Ontario, Canada, and the United States. J. Clin. Oncol. 17(7), 2244-2255 (1999).

16 Hershman D, McBride R, Jacobson JS et al. Racial disparities in treatment and survival among women with early-stage breast cancer. J. Clin. Oncol. 23(27), 6639-6646 (2005).

17 Pearcey R, Miao Q, Kong W, ZhangSalomons J, Mackillop WJ. Impact of adoption of chemoradiotherapy on the outcome of cervical cancer in Ontario: results of a population-based cohort study. J. Clin. Oncol. 25(17), 2383-8 (2007).

18 Booth CM. Evaluating patient-centered outcomes in the randomized controlled trial and beyond: informing the future with lessons from the past. Clin. Cancer Res. 16(24), 5963-5971 (2010).

- Importance of integration between randomized clinical trials and large database approach.

19 Sanoff HK, Carpenter WR, Stürmer T et al. Effect of adjuvant chemotherapy on survival of patients with stage III colon cancer diagnosed after age 75 years. J. Clin. Oncol. 30 (21), 2624-2634 (2012).

20 Cheung WY, Shi Q, O'Connell M et al. The predictive and prognostic value of sex in early-stage colon cancer: a pooled analysis of 33,345 patients from the ACCENT database. Clin. Colorectal Cancer 12(3), 179-187 (2013).

21 Lieu CH, Renfro LA, de Gramont A et al. Association of age with survival in patients with metastatic colorectal cancer: analysis from the ARCAD Clinical Trials Program. J. Clin. Oncol. doi:10.1200/JCO.2013.54.9329 (2014) (Epub ahead of print).

22 Valentini V, van Stiphout RGPM, Lammering G et al. Nomograms for predicting local recurrence, distant metastases, and overall survival for patients with locally advanced rectal cancer on the basis of European randomized clinical trials. J. Clin. Oncol. 29(23), 3163-3172 (2011).

-. Initial result of validity and usefulness of predictive models in rectal cancer.

23 Valentini V, Aristei C, Glimelius B et al. Multidisciplinary rectal cancer management: 2nd European Rectal Cancer Consensus Conference (EURECA-CC2). Radiother. Oncol. 92(2), 148-163 (2009).

24 Haustermans K, Debucquoy A, Lambrecht M. The ESTRO Breur Lecture 2010: toward a tailored patient approach in rectal cancer. Radiother. Oncol. 100(1), 15-21 (2011).

25 Van den Bogaard J, Janssen MHM, Janssens $\mathrm{G}$ et al. Residual metabolic tumor activity after chemo-radiotherapy is mainly located in initially high FDG uptake areas in rectal cancer. Radiother. Oncol. 99(2), 137-141 (2011).

26 Lambin P, Roelofs E, Reymen B et al. "Rapid Learning health care in oncology" - an approach towards decision support systems enabling customised radiotherapy'. Radiother. Oncol. 109(1), 159-164 (2013).

- Personalized medicine is useful in choosing the optimal treatment for each patient redirecting the right resource to the right patient.

27 Vickers AJ. Prediction models: revolutionary in principle, but do they do more good than harm? J. Clin. Oncol. 29(22), 2951-2952 (2011).

28 (AJCC) AJC on C. AJCC 7th Edition - Colon and Rectum Cancer Staging (2007). https://cancerstaging.org

29 Sobin L, Parkin DM. International Classifi cation of Diseases for Oncology. Third Edition. World Health Organization, Geneva, Switzerland. http://whqlibdoc.who.int

30 CTEP. Common Terminology Criteria for Adverse Events v3.0 (CTCAE) (2006). http://ctep.cancer.gov

31 Services H. Common Terminology Criteria for Adverse Events (CTCAE) (2010). http://evs.nci.nih.gov

32 Kumar V, Gu Y, Basu S et al. Radiomics: the process and the challenges. Magn. Reson. Imaging 30(9), 1234-1248 (2012).

33 Valentini V, Schmoll HJ, Velde CJ Van De. Multidisciplinary Management of Rectal Cancer. Springer-Verlag Berlin, Heidelberg, Germany (2012). 
34 Lewalle P, Rodrigues JM, Zanstra P et al. A deployment and Research Roadmap for Semantic Interoperability: the EU semantic health project. Stud. Health Technol. Inform. 136, 635-640 (2008).

35 Roelofs E, Dekker A, Meldolesi E, van Stiphout RGPM, Valentini V, Lambin P. International data-sharing for radiotherapy research: an open-source based infrastructure for multicentric clinical data mining. Radiother. Oncol. 110(2), 370-374 (2014).

36 Maastricht Radiation Oncology. Validation of a predictive model after complete response in rectal cancer (Thunder). ClinicalTrials.gov Identifier:NCT00969657. www.clinicaltrials.gov/show/NCT00969657

37 Jayasurya K, Fung G, Yu S et al. Comparison of Bayesian network and support vector machine models for two-year survival prediction in lung cancer patients treated with radiotherapy. Med. Phys. 37(4), 1401-1407 (2010).

38 Dehing-Oberije C, Yu S, De Ruysscher D et al. Development and external validation of prognostic model for 2-year survival of non-small-cell lung cancer patients treated with chemoradiotherapy. Int. J. Radiat. Oncol. Biol. Phys. 74(2), 355-62 (2009).

39 MAASTRO Clinic. MAASTRO prediction website. www.predictcancer.org

40 Recommendation WWWC. Resource Description Framework (RDF) Model and Syntax Specification (1999). www.w3.org

41 Assélé Kama A, Choquet R, Mels G, Daniel C, Charlet J, Jaulent M-C. An ontological approach for the exploitation of clinical data. Stud. Health Technol. Inform. 192, 142-146 (2013).

42 Meldolesi E, van Soest J, Dinapoli N, et al. An umbrella protocol for standardized data collection $(S D C)$ in rectal cancer: a prospective uniform naming and procedure convention to support personalized medicine. Radiother. Oncol.. 112(1), 59-62 (2014).

43 Semantic Web platform. http://tinyurl.com/VateSPARQL1

44 National Cancer Institute: Comprehensive Cancer Information at the National Institute of Health. www.ncicb.nci.nih.gov

45 Luta G, Ford MB, Bondy M, Shields PG Stamey JD. Bayesian sensitivity analysis methods to evaluate bias due to misclassification and missing data using informative priors and external validation data. Cancer Epidemiol. 37(2), 121-126 (2013).

46 Marsolo K. Approaches to facilitate institutional review board approval of multicenter research studies. Med. Care 50(Suppl. 7), S77-S81 (2012).

47 Boyd S. Distributed optimization and statistical learning via the alternating direction method of multipliers. Found. Trends Mach. Learn. 3(1), 1-122 (2010).

48 Harrel F. Resampling, Validating, Describing, and Simplifying the Model. Springer-Verlag, NY, USA, 87-103 (2001).

49 Oh JH, Craft J, Al Lozi R et al. A Bayesian network approach for modeling local failure in lung cancer. Phys. Med. Biol. 56(6), 1635-1651 (2011).

50 Van Stiphout RGPM, Lammering G, Buijsen $\mathrm{J}$ et al. Development and external validation of a predictive model for pathological complete response of rectal cancer patients including sequential PET-CT imaging. Radiother. Oncol. 98(1), 126-133 (2011).

-. Initial result of validity and usefulness of predictive models in rectal cancer.

51 Vickers AJ, Cronin AM, Elkin EB, Gonen M. Extensions to decision curve analysis, a novel method for evaluating diagnostic tests, prediction models and molecular markers. BMC Med. Inform. Decis. Mak. 8, 53 (2008).

52 Vickers AJ, Elkin EB. Decision curve analysis: a novel method for evaluating prediction models. Med. Decis. Making 26(6), 565-574 (2006)

53 Wu Y, Jiang X, Kim J, Ohno-Machado L. Grid Binary LOgistic REgression (GLORE): building shared models without sharing data. J. Am. Med. Inform. Assoc. 19(5), 758-764 (2012).

54 Liu K, Kargupta H, Member S, Ryan J. Random projection-based multiplicative data perturbation for privacy preserving distributed data mining. IEEE Trans. Knowl. Data Eng. 18(1), 92-106 (2006).

55 Valentini V, Lambin P, Myerson RJ. Is it time for tailored treatment of rectal cancer? From prescribing by consensus to prescribing by numbers. Radiother. Oncol. 102(1), 1-3 (2012).

- It enhances the necessity to go into the direction of a personalized medicine. 